\title{
Ueber Kopfhöhlenrudimente beim Menschen.
}

\author{
Von \\ K. W. Zimmermann, \\ a. o. Professor der Anatomie in Bern. \\ Hierzu Tafel XXII.
}

Als ich an einem menschlichen Embryo von 3,5 mm N.St.- $\mathrm{L}^{1}$ ) die Blutgefässe des Kopfes untersuchte, fielen mir auf der rechten Seite lateral von der Carotis interna und zugleich lateral von der Anlage der Rathkeschen Tasche etwas hinter der Augenblase in einer zellreicheren Gegend des Mesoderms, nahe dem Mundhöhlenepithel, drei s ch arf begrenzte kleine L umina (s. Fig. 2) auf, von denen das vordere (ventrale) am grössten und etwas in die Länge gezogen (a), das hintere (dorsale) am kleinsten (c) erschien. Das mittlere (b) und das hintere waren rundlich. Erst dachte ich an Querschnitte von Blutgefässen, ein genaueres Studium der traglichen Gebilde bei stärkerer Vergrösserung und die Verfolgung derselben durch die verschiedenen Schnitte hindurch belehrte mich jedoch bald eines Besseren. Zunächst constatirte ich, dass die Lumina allseits geschlossenen Bläschen angehörten. Die Wände derselben waren von epithelartigen Zellen begrenzt, welche peripher spitz ausliefen. Der an der Bildung des Lumens betheiligte Abschnitt dieser Zellen war im Allgemeinen etwas breiter, doch wechselte die Breite und Länge bei den einzelnen Zellen sehr. Die Kerne lagen zum Theil in gleicher Höhe, doch fanden sich auch verschiedene aus der Kernreihe mehr oder weniger peripheriewärts herausgerückt. Dieselben gehörten schmäleren und längeren Zellen

1) Kurz vor dem Einbetten gemessen. Der Embryo entspricht äusserlich ganz dem von Rabl einer Selbstmörderin 8 Stunden post mortem entnommenen $4 \mathrm{~mm}$ langen Embryo. Der Erhaltungszustand ist zwar nicht der allerbeste, da das Hirnrohr stark unregelmässig gefaltet ist, doch sind die hier in Betracht kommenden Verhältnisse durchaus gut erhalten und klar zu erkennen, so dass es ausgeschlossen erscheint, dass die zu beschreibenden Gebilde etwa Kunstprodukte seien. 
an. Mehrere weiter abliegende Zellen sandten noch einen ganz schmalen Fortsatz zwischen die ïbrigen Zellen hinein bis zum Lumen. Wieder andere von rein spindelförmiger Gestalt liessen' den entspsechenden Fortsatz zwar \%wischen die das Lumen begrenzenden Zellen hinein, aber nicht mehr bis zum Lumen selbst verfolgen. Alle drei Höhlen zeigten den gleichen Charakter und waren nur durch die Weite der Lumina verschieden.

Als ich durch Verfolgen der Schnitte erkannt hatte, dass es sich um geschlossene Bläschen und nicht um Querschnitte von Kanälchen handelte, drängte sich mir mit Rücksicht auf die Lage und auf den Bau der Gebilde der Gedanke auf, dass es sich um kopfhöhlenähnliche Bildungen handelte. Ausschlaggebend wurde der Umstand, dass i ch a u c h a uf der linken Seite genau an derselben Stelle ganz äbnliche Verhältnisse aufzufinden vermochte, ferner dass bei Selachiern in derselben Gegend Augenmuskeln bildende Kopfböhlen liegen, und dass bei einem menschlichen Embryo von $7 \mathrm{~mm}$ Länge in der gleichen Gegend ein grösserer, zellreicherer Bezirk sich vorfand, in welchem zwar keine Spur von Kopfhöhlen mehr nachweisbar war, in dem aber der Oculomotorius, Trochlearis und Abducens sich verloren, so dass ich annehmen musste, die die Augenmuskeln liefernden Zellmassen seien aus den fraglichen Gebilden hervorgegangen.

Nun lag der Gedanke nahe, dass in den drei kleinen Höhlen die drei entsprechenden Kopfhöhlen der Selachier zu suchen seien, aus denen sich die Augenmuskeln entwickeln, dass also die vorderste grosse Höhle die Oculomotoriusmuskulatur, die mittlere den M. obliquus superior, die hintere den M. rectus lateralis liefere. Diese Erwägung wurde aber durch den Befund auf der linken Seite zu nichte gemacht, da sich hier.statt der drei im Ganzen siebenkleinere Höhlen fand en und zwar zwei grössere, drei mitlere und zwei kleinere. Dieser Befund konnte zwar an der Deutung der Gebilde als Kopfhöhlen nichts ändern, machte es jedoch zur Gewissbeit, dass man nicht etwa in jeder der Kopfhöhlen eine einer bestimmten der Selachier entsprechende ror sich habe, wenigstens nicht auf der linken Seite, dass man vielmebr in ihnen nur $R$ este ron Kopf- 
höhle n zu sehen habe. Ob von einer oder von allen dreien, lässt sich noch nicht entscheiden.

Noch ist zu bemerken, dass irgend welche röhrenförmige Reste in den Visceralbogen nicht aufzufinden waren.

Ich babe auch einige andere Säugethierembryonen entsprechenden Alters auf Kopfhöhlenrudimente hin untersucht, jedoch bei keinem auch nur eine Andeutung von solchen gefunden, womit ich jedoch durchaus nicht sagen möchte, dass dergleichen nur beim Menschen vorkäme. Vielleicht existiren die Bildungen nur kurze Zeit, und es standen mir nicht die richtigen Stadien zur Verfügung, oder das Auftreten derselben. ist individuellen Schwankungen unterworfen ${ }^{1}$ ). Es wäre sehr wünschenswerth, dass die Herren Fachgenossen, welche über eine grössere Zahl von Säugethierschnittserien verfïgen, dieselben auf Kopfhöhlenrudimente hin untersuchten. Ich bin überzeagt, dass wir dann bald über das Verhalten derselben bei den Säugern im Allgemeinen ins Klare kommen werden.

Zum Schluss gebe ich noch einige Maasse der Höhlenlumina. $\mathrm{Da}$ die äusseren Contouren der Gebilde, wie die Fig. 2 zeigt, keine bestimmten sind, so lassen sich auch genaue Zahlen für die Gesammtgrösse nicht angeben.

Rechts (drei Höhlen. Sie lagen annähernd in der gleichen Sagittalebene):

\begin{tabular}{|c|c|c|c|c|c|}
\hline & grösster & Läng:sdurchmesser & des & Lumens: & $43 \mu$ \\
\hline a. & & Querdurchmesser & $n$ & $\pi$ & $19 \mu$ \\
\hline & grösster & Durchmesser & & $\pi$ & $16 \mu$ \\
\hline & kleinster & $n$ & $\pi$ & $\pi$ & $12 \mu$ \\
\hline & grösster & $\pi$ & $?$ & $\pi$ & $9 \mu$ \\
\hline & kleinster & $"$ & & $\pi$ & 6 \\
\hline
\end{tabular}

Links (sieben Höhlen. Die Reihenfolge der Buchstaben dentet an, wie die Gebilde ron innen nach aussen aufeinander folgen; e und f lagen im gleichen Schnitt):
a.
Durchmesser des Lumens
$9 \mu$
b.

$\begin{array}{llll}n & n & n & 18 \mu \\ n & n & n & 23,8 \mu \\ n & n & n & 13,4 \mu\end{array}$

1) Ich besitze einen Eidechsenembryo, bei dem eine Höhle auf der einen Körperhälfte sehr schön entwickelt ist, auf der anderen aber vollständig fehlt und durch eine zellreichere Stelle vertreten wird. 
484 K. W. Zimmermann: Ueber Kopf höhlenrudimente b. Menschen.
d.
Durchmesser des'Lumens $\quad 6 \mu$
e. $\left\{\begin{array}{l}\text { glösster } \\ \text { kleinster }\end{array}\right.$

$\pi$
$\pi$
$n$
$"$
$18 \mu$
f. $\left\{\begin{array}{l}\text { grösster } \\ \text { kleinster }\end{array}\right.$
g.
$n$ n $n$

$4,4 \mu$
$15 \mu$
$6 \mu$
$5,5 \mu$

B e r n, den 10. Oktober 1898.

\section{Erklärung der Abbildungen anf Tafel XXII.}

Fig. 1. Sagittalschnitt eines menschlichen Embryo von $3,5 \mathrm{~mm}$ N.St.-L., die drei Kopfhöhlenrudimente der rechten Seite enthaltend: 1. 2. 3. 4 erster, $\mathrm{zweiter}$, dritter, vierter Visceralbogen. A. Aorta; Al. Allantois; C. Herz: $D, D, D$ Darm; $G$. Gehörbläschen; $H$. Leber; $K$. die drei Kopfhöhlenrudimente; $L$. Leibeshöhle; $M, M, M$. Medullarrohr; $N$. Nabelvene; $P$. rechte Lungenanlage; $S$. Schwanzdarm; $V$. Vorderhirn. Vergr. 26 fach.

Fig. 2. Die drei Kopfhöhlen der rechten Seite (a. b. c). M. Mundhöhlenepithel. Vergr. 490 fach.

Fig. 3. Die sieben Kopfhöhlen der linken Seite (a bis $g^{\prime}$; nur Umrisse). 1. erster Visceralbogen; A. Augenanlage. Vergr. 168 fach. 